to the contents of watch glass No. 1 it is obvious that this pipette cannot be used to mix the contents of the watch glass without being re-sterilised, as it has been soiled for a considerable portion of its length with a fluid containing spore-bearing organisms.) The first pipette is then laid aside and another one is taken in hand. 'The contents of the second watch glass are thoroughly mixed and 100 cubic millimetres and ten cubic millimetres are planted out in the tubes numbered 5 and 6 . From the 1 in 10,000 dilution five cubic millimetres are transferred to the third watch glass giving a dilution of 1 in $1,000,000$. The third pipette is now taken in hand and after thorough mixing 100 cubic millimetres and ten cubic millimetres of this dilution are planted out in the tubes numbered 7 and 8 . The whole of the process described can, of course, be carried out with the aid of a single pipette, provided it be sterilised thoroughly in the flame between each dilution. This, however, occasions considerable loss of time and for rapid working three pipettes will be found essential. The remaining steps of the technique will vary according to the wishes of the investigator. Taking either the gelatin or agar series of tubes the quantity of water (in cubic centimetres) planted out will be as follows : tube $1,0.1$; tube $2,0.01$; tube $3,0.001$; tube 4 , 0.0001 ; tube $5,0.00001$; tube $6,0.000001$; tube 7 , 0.0000001 ; and tube 8,0.00000001. If it is only desired to ascertain the limits within which the number of organisms per cubic centimetre must lie then the tubes are simply incubated in the vertical position and the results are interpretated as follows. Growth in tube 1, but not in tube 2, would place the number of organisms per cubic centimetre between 10 and 100 . Growth in tube 2 , but not in tube 3 , would place the number of organisms per cubic centimetre between 100 and 1000 , and so on, the limits adopted being those in general use for the classification of waters by the number of contained organisms. If it is desired to know the actual number of organisms in terms of $10,100,1000$, \&c., it will be necessary to distribute the water planted out over the surface of the medium and to incubate the tubes in the sloping position in order to prevent the accumulation of organisms at the foot of the slope. If preferred the quantity of water planted out may be spread over the surface of the medium with a glass rod but in actual practice $I$ have found this to be unnecessary as in the case of the larger quantities even distribution can be obtained by manipulation of the tube, whilst in the case of the smaller quantities sufficient distribution can be obtained by means of the pipette at the time of planting out. The following three examples will indicate sufficiently well the results obtainable by this method:-

\section{Sample $A$.}

Gelatin at $22^{\circ} \mathrm{C}$. for seven days :- $\quad$ Agar at $37^{\circ} \mathrm{C}$. for 48 hours :-

Tube 1. Growth (liquefied). Tube 1. Growth.

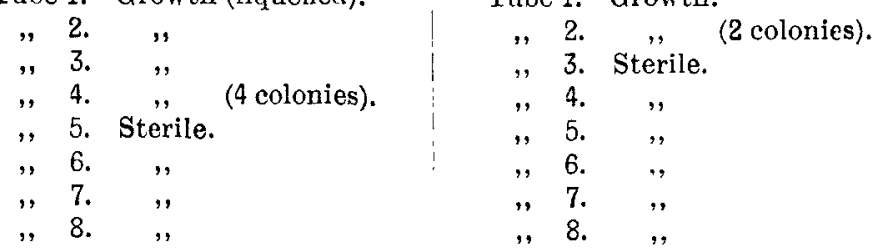

Number of organisms per cubic centimetre of gelatin at $22^{\circ}$ C., 40,000 .

" " " , agar at $37^{\circ} \mathrm{C}$., 200 .

2. Sample $B$

Agar at $22^{\circ} \mathrm{C}$. for seven days :- i Agar at $37^{\circ} \mathrm{C}$, for 48 hours:Tube 1. Growth.

"2. "

"3. " " (1 colony).

Tube 1. Growth.

"4. Sterile

,2. ", (14 colonies)

,3. ", (1 colony).

, 5. , ,

$\Rightarrow 6 . \quad " \quad 7$

, $8 . \quad$,

Number of organisms per cubic centimetre of agar at $22^{\circ} \mathrm{C} ., 1000$.

"between tubes 2 and 3).

$37^{\circ} \mathrm{C} ., 1200$ (mean

3. Scomple C.

Agar at $22^{\circ} \mathrm{C}$. for seven days :-

\begin{tabular}{l|l} 
Tube 1. Growth. Tube 5. Sterile. &
\end{tabular}

, 2.

, 3.

(5 colonies).

Tube 5. Sterile.

, 7

", 8.

Number of organisms per cubic centimetre of agar at $22^{\circ} \mathrm{C}, 50,000$. effluents one pipette will be sufficient as washing out with boiling water between the dilutions will meet the requirements of the case and the pipette can then be rapidly cooled by washing out with sterile water. With regard to bacillus coli, \&c., the special media and methods of incubation used in such cases will, of course, take the place of the ordinary media and methods of incubation used in the mere enumera. tion of organisms in water.

In conclusion it will be well to consider the advantages and disadvantages of this technique as compared with the plating-out method. The following disadvantages may be urged against the technique :-

1. The diluting pipette being home-made is liable to be inaccurate. I have had a batch of these pipettes tested against a standard one centimetre pipette and it was found that while the relative error as between pipettes was barely appreciable the absolute error in one centimetre was in all cases less than $0 \cdot 01$ centimetre.

2 . The method does not admit of microscopic examination of the colonies which develop. This difficulty can easily be overcome by subculture.

Against these disadvantages the following advantages may be set forth :-

1. It relieves the investigator of all anxiety on the score of the limit dilution to be employed when dealing with unknown waters, \&c. By the employment of this technique all unpleasant surprises can be avoided. Whether the sample contain 12 organisms per cubic centimetre, or $12,000,000$, the number will be estimated with equal accuracy and beyond the possibility of mishaps, such as "rapid liquefaction of the plates," "organisms innumerable in a thousandfold dilution."

2. The technique permits of very rapid working. With the aid of an ordinary coolie I have found that the whole process takes between six and seven minutes.

3. The small quantity of apparatus required.

4. There is practically no waste in this method as all tubes which remain sterile are ready for use again.

5. The pipettes are easily made, inexpensive, and can be sterilised in the flame without fear of breakage.

6 . The whole process can be carried out at the water side, a spirit lamp being added to the armamentarium for the purpose of sterilising the pipette.

7. The labour of counting colonies in a Petri dish is entirely dispensed with and consequently all artificial aids to counting, such as counting discs, cease to be necessary.

Kasauli, India.

\section{SEVEN CASES OF BERI-BERI.}

By P. N. GERRARD, M.D. DURH., D.T.M.H. CANTAB., DISTRICT SCRGEON, KRIAI, FEDERATED MALAY STATES,

Sixce my return to the East I have had the opportunity of observing and of carrying out post-mortem examinations upon seven fulminating cases of beri-beri which have occurred in the District Hospital, Taiping. As some of the observations which have been made are of interest and as the disease has of late attracted a certain amount of attention at home it seems to me advisable to publish these cases to the profession in view of the fact that fulminating cases occurring under the conditions of a local epidemic are rare. The cases all occurred in the same ward. All the cases except one occurred in patients who had been for a considerable time under treatment for beri-beri and the one exception was a case which was brought to hospital in the fulminating state but late in the local epidemic, which lasted about one month. All of the patients in the other wards of the hospital, 400 in number, were receiving similar diets to those which were prescribed for the subjects of the cases which fulminated and no patient in the ward where the epidemic occurred who was not admitted in the first instance for beri-beri acquired the disease. (There may be one exception to this statement in the case of a Chinese, aged 38 years, who was admitted on Oct. 17th, 1904, for dysentery. He recovered from his dysentery but developed beri-beri which was diagnosed as such on Dec. 3rd.) The majority of the patients in the ward who were not suffering from beri-beri were phthisical. One end of this ward was

In the quantitative estimation of bacillus coli in sewage used as a reception ward for all cases that were admitted to 
the hospital. In the reception ward the patients remain for about 48 hours and are then transferred to the wards set apart for their respective diseases. No beri-beri has occurred amongst these patients.

CASE 1.-The first case occurred in a Chinese, 53 jears of age, who was admitted on August 21st, 1904. He had been nine years in the country and gave a history of having suffered from a disease which was apparently beri-beri for six months. His first bad symptoms occurred on Nov. 14th and he died on the 18th. The treatment of his case was on general principles and no attempt was made to discover the gastric reaction, favourable or unfavourable, to the poison (if such it is) as was done in subsequent cases. He had an iron and arsenic mixture to start with and subsequently, on the increase of his oetema, he was put on a diuretic and when the heart commenced to show signs of failing digitalis was added (Nov. 5th). There were no ankylostoma ova in the stools and until the 14th nothing to excite suspicion of impending crisis in the case arose. On the 14th his pulse was $100^{\circ} \mathrm{F}$. and his respirations were 30 . He had a pericardial friction rub and the case commenced to fulminate; artificial serum clysters were given twice daily without any relief to the symptoms. The patient died on the 18th at 11 P.M. - that is, three months after his admission to hospital, 96 hours from the commencement of fulmination, and nine months from the commencement of his disease, according to his history.

A posi-mortem exanination was performed on the day of the patient's death and the most important observation in this case was as usual in connexion with the stomach and intestines. The stomach was dilated and congested with deep purple erosions for a considerable area in the neigh. buurhood of the pylorus It contained a mucoid fluid in which a bacillus $2.8 \mu$ long by $1.05 \mu$ predominated (Hamilton Wright's bacillus measured from 4 to $6 \mu$ by from 1 to $1.5 \mu)$.

CASE 2.-The patient in this case was admitted to hospital on July 15th, 1904. He gave a history of having been ill for two months before admission. He was 25 years of age and had been in the country for three years. During the early days of his stay in hospital he was treated in the usual routine manner with mixtures of iron combined with strychnine and arsenic. On Nov. 21st fulminating symptoms appeared and solution of trinitrin was administered with the object of relieving the right heart; the stomach symptoms were severe, pain and fulness being both complained of, the latter being evidenced by the usual epigastric inflation and puffiness. With a view to alleviating the gastric distress the stomach was washed out naturally, one and a half pints of warm water being given by the mouth after the exhibition of 30 grains of ipecacuanha powder. In view of the possibility of the disease being due to the absorption of a toxin from the alimentary tract the patient was put on a 1 per cent. solution of formalin by the mouth, 15 minims every three hours in water. This proved of no avail; the patient only lived to receive four doses and he died at $1 \mathrm{~A}$.M. on the 22nd.

A point worthy of mentioning in this case is that one of the first symptoms which interrupted the course of what might be described as a chronic case and heralded the fulmination was an extreme tenderness over the splenic area. The abdomen generally was somewhat painful on deep pressure and all the abdominal muscular reflexes were greatly increased. The spleen, however, remained normal in size. The fæcal excretion remained normal tbroughout; the urine, however, was as usual diminished.

The post-mortem examination, made on the afternoon of the 22nd, showed the following conditions: there was deep congestion of all the mucous membranes, an ounce of fluid was present in each pleura, there was deep general congestion of the lungs, the pulmonary artery was full of dark clot, the pericardium contained four ounces of fluid, and the anterior surface of the heart showed punctiform hæmorrhages. All the other organs were congested and the stomach exhibited a dull reddish-purple interior with considerable erosion which was most marked at the pylorus.

CASE 3.- The patient, a Chinese, aged 23 years, living in the town of Taiping, was admitted into hospital on Nov. Ist, 1904. He gave a history of having been ill for two months. He had been eight months in the country. He was treated at first on ordinary lines-iron preparations, \&c. On the 1 Studies from the Institute for Medical Research, Federated Malay
States, vol. ii., No. 2 . appearance of fulminating symptoms, however, which set in on the 21st, I put him on permanganate potassium pills (one grain) morning and evening. Subsequently trinitrin and strychnine had to be exhibited and nitrite of ampl inhalations to be employed. A mustard plaster was also applied over the heart on account of the presence of pericardial fluid. The patient died at 9 A.M. on the $22 n d$.

The necropsy showed deep congestion of the mucous membranes and of the nails and finger-tips, about an ounce of fluid in each pleura, deep congestions of the lungs, cyanotic enlargement of the liver and the spleen, four ounces of fluid in the pericardium, and the usual severe erosion of the pylorus which extended down the jejunum. This patient had only four grains of permanganate of potassium from the time when it was first exhibited until he died; this was hardly sufficient to expect any rapid result from.

CASE 4.-The patient was a man, aged 45 years, who had lived for 18 years in the country. $\mathrm{He}$ was admitted to hospital on Oct. 25th, 1904. Fulminating symptoms showed themselves first on Nov. 24th. At 10.30 A.M. on this day he was in such distress with epigastric pain and dyspncea that I tried an emetic of ipecacuanha powder followed by warm water in quantity but no emesis took place and gastric paralysis had probably already taken place. At 11.30 A.M. his symptoms became alarming and the engorgement of the right heart clear, so he was bled from the median basilic to ten ounces with relief for about an hour. Strychnine was exhibited and a stimulating mixture of digitalis was administered frequently. The patient died in deep distress at 3.20 P.M. on the same day, the illness having run a very rapid course.

With regard to the post-mortem appearances, there was no rigor mortis such as I have frequently observed in beri-beri cases. Both lungs were intimately "glued" to the chest wall throughout their whole surface, presumably due to acute emphysema, due in turn to a paralytic state of the air vesicles probably, the pericardium contained two and a half ounces of fluid, the heart was enormous. and the surface veins were engorged and dilated. The stomach showed the usual purple erosion, which was most marked at the cardiac end in this case; the condition increased in severity towards the lower end of the duodenum.

A differential leucocyte count in this case at the commencement of fulmination showed: polymorphonuclears, $53 \cdot 3$ per cent. ; large mononuclears, $7 \cdot 3$ per cent. ; lymphocytes, 33.3 per cent.; eosinophiles, 1.6 per cent.; and transitionals, $4 \cdot 3$ per cent. No malaria parasites were observed.

CASE 5.-The patient, a man, aged 29 years, who had lived for five years in the country and who had been ailing for 20 days, was admitted to hospital on Nov. 24th, 19c4. Fulminating symptoms occurred on the 28th and death tnok place at 9.50 P.M. on the 30 th, the crisis having lasted 50 hours. On the 25 th he was placed upon a mixture containing creasote and iodoform which I have been giving as a routine mixture in the treatment of beri-beri, with the object of checking gastric fermentation and attempting to heal the erosion which occurs in all these cases. He was also given small doses of a cardiac stimulant and a sudorific. On the 28th he refused to take his medicine, saying that it made him feel "worse," and his obstinacy was so great that although other patients in the ward expressed themselves as "feeling better" on the same mixture I changed it in his case to a stimulat ing mixture containing carbonate of ammonia, digitalis, and nux vomica. On the 29th he was so ill with distressed breathing and all the usual signs of cardiac engorgement that I commenced clysters of normal saline solution with the object of preventing clotting in the right ventricle and. if possible, of diluting the unknown poison in the blood. On this date the reaction of his fæces was highly acid and vomiting started. Saline enemata (six ounces every three hours) were persisted in without amelioration of his condition and on the morning of his death-namely, the 30th-five days after the premonitory symptoms of crisis (namely, dyspncea, epigastric pain, fulness, and tenderness, and increase of the cedema, with vomiting), the case assuming a dangerous aspect, he was placed on solution of trinitrin and strychnine injections were administer $d$. The following was his physical condition on that date. The pulse was 128 , the respirations were 42 , the temperature was $99 \cdot 8^{\circ} \mathrm{F}$., the liver was two and a half inches below the costal margin, the spleen was not palpable, and pretibial cdema was increasing. The amount of urine voided in 24 hours was 27 ounces. As the bowels had be en constipated 
for tiwo days the patient was given as a forlorn hope one minim of croton oil. The vomiting, however, continued and death took place at 9.50 P.M.

Post mortem marked rigidity was met with, the finger tips and lips were livid, in the right pleura wero found seven ounces of fluid and in the left three and a half' ounces, the pericardium contained 16 ounces of fluid which was not diagnosable by percussion 12 hours before death (probably a fulminating hydro-pericarditis-compare the acute empliysema mentioned above), the gall-bladder was distended, the liver was congested, enlarged, and somewhat friable, the spleer was deeply congested, enlarged, and friable, and the kidneys were congested. The erosion was not so marked in the stomach itself, but the pylorus and the first stage of the duodenum were very severely affected. In the intestines patches of erosion occurred with intervals of healthy intestine of from six inches to one and a half inches in length; these erosions occurred throughout the small intestine, and at longer intervals in the large intestine right up to the anus. There was also a subacute appendicitis in this case.

CASE 6.-The patient, a man, aged 43 years, who had lived in this country for 20 years, and who had been ailing for 10 days, was admitted in a condition of pneumogastric crisis on Dec. 4th at 11.30 A.M. He died at 1.15 P.M. on the same day: Nothing could be done for this man as he was nearing dissolution on arrival. I quote the case merely to complete my series.

Post-mortem examination showed rigidity to be only fairly marked. The face, neck, fingers, and lips were almost black. The pleura and lungs were normal, in the pericardium were two and a half ounces of fluid, the heart was large but healthy, there was commencing cirrhosis in the liver, the gall-bladder was full and tense, the spleen weighed one pound and was congested, and the kidneys were congested. There were hæmorrhagic patches about the middle and at the pyloric end of the stomach; these also occurred at the duodenum and intermittently also in the jejunum, becoming more intense towards the lower end; they were very numerous and well marked in the ileum. The cæcum was congested. The first part of the colon was healthy but the erosions occurred again on approaching the lower end as far as the rectum, which in itself was healthy.

CASE 7.- The patient was a Chinese, aged 29 years. $\mathrm{He}$ had been resident for a year in the Federated Malay States. The duration of the disease was said to have been one month. He was admitted to hospital on Sept. 20th, 1904 ; fulminating symptoms developed on the morning of Dec. 23rd and death occurred on the same day. This patient was first placed on a mixture of iron and quassia. On Oct. 7 th a stimulating mixture containing carbonate of ammonia and digitalis was exhibited. This proved of no practical benefit to the patient and with a view to determining whether the toxin was more partial to an alkaline or acid reaction in the stomach I put him on a strongly alkaline mixture of soda and carbonate of ammonia on Nov. 29th. The secretion of urine throughout this case was very small, the daily average for the six days commencing Nov, 29th being $12 \cdot 16$ ounces; it contained no albumin but there was a copious deposit of phosphates. On Dec. 8 th the urine had diminished to six ounces in 24 hours; he was given a mixture containing iodide of potassium and digitalis and a hot bath twice daily. The stools were examined for ankylostoma ova but without finding any. On the 21st the cedema had become more marked and the general condition of the patient was worse. On the morning of the 23 rd the temperature rose to $100 \cdot 6^{\circ} \mathrm{F}$, the cedema was much more severe, and the crisis commenced. The urine averaged for the 15 days previous to this date 8.5 ounces in 24 liours. I made prolonged efforts to save this patient. He was bled to relieve the right heart, the pericardium was tapped to relieve the effusion, and transfusion of saline solution was pushed, but to no purpose, and he died on the operating table with the cannula still in his median basilic vein.

With regard to the tapping of the pericardium in this case I may admit freely that there was more cardiac dilatation than fluid in question and I will also remark that the right ventricle was wounded superficially by $\mathrm{my}$ trocar. I would therefore recommend that in future where patients suffering from beri-beri are concerned (in whom the cardiac dilatation may be, and often is, beyond all anticipation in its extent) if the pericardium is to be tapped let it be by means of deliberate operation, in which the membrane is to be picked up and carefully punctured in a direction clear of the enormously dilated heart. I would draw attention to the following points in the history of this case: (1) that the urine during the last 15 days of life was less than the norma amount by 622 ounoes; (2) that the temperature rose at the commencement of the crisis ; and (3) that alkaline mixture brought about no improvement.

A post-mortem examination was made within ten minutes after the death of the patient. Rigidity was not yet present, the skin and tissues were very cedematous, the muscles were deeply congested, the superficial veins on the stomach were injected, and the body generally was anasarcous. In the right pleura a small amount of fluid was met with and in the pericardium five and a half ounces of blood-stained fluid had collected. The heart was enormously dilated and flabby (one of the most striking cases of paralytic "jelly-fish dilatation," if I may coin a term, which I have seen); a small puncture on the left ventricle did not penetrate. The liver was congested, normal in size, and the gall-bladder was full (paralysed ?) ; the spleen was slightly enlarged and soft; the right and left kidneys were small and very hard but were apparently normal ; the capsules stripped easily; the stomach was small and the mucous membrane was reddened diffusely and considerably thickened ; in the intestines there' were two patches of ill-marked inflammation in the second and third stages of the duodenum and two round worms' were found lower down ; and there was a patch of marked inflammation in the upper part of the descending porition of the colon.

In concluding these cases I' would remark that in themselves they are instructive in many ways and may, I think I can safely say, add to our knowledge of fulminating beriberi to some extent: The following points are, I consider, worthy of note as evidence additional to the well-known classical signs. 1. That the commencement of crisis' is: sometimes marked (a) by a rise of temperature; (b) by an increase of abdominal reflexes'; $(c)$ by splenic tenderness and to some extent general abdominal tenderness; $(d)$ by diminution in the quantity of urine passed; and $(e)$ by congestion of the mucous membranes. 2. That alkalies favour the development of the disease. Although onlyone of these cases exemplifies this point, I have, I believe, proved it in many others. 3. That no dilution of the poison in the blood by means of saline clysters or transfusion is possible and that the crisis is, therefore, in all probability a collapse of the nervous system, and the poison may be transmitted purely through nerve tissue. 4. That bleeding is of no permanent value (previously established, I consider). 5. That no alteration of the normal proportions of the leucocytes occurs.

The hospital beds are infested with bugs which arealmost impossible to exterminate, owing to faulty construction and the Perak system of blanket bed-covers instead of mats: I have examined microscopically' several bugs from the be'ds' of fulminating cases but have found nothing suspicious. I must express my regret that my impending transfer to a district where there is practically no beri-beri will probably interrupt for some considerable time my further remarks upon this disease. I have been obliged thereby to publish these cases in a somewhat rough form but trust that they may be of interest.

Krian. Federated Malay States.

A FATAL CASE OF PRECIPITATE LABOUR:

\section{BY H. E. RAYNER, F.R.C.S. ENG., L.R.C.P. LOND.,} $A \times D$

\section{W. L. STUART, M.D., B.S. LOND., M.R.C.S. ENG., L.R.C.P. LOND.}

FATAL cases of precipitate labour comparable with the following are not unknown, but fortunately they appear to be of sufficiently rare occurrence to justify the publica. tion of this case, which, though it may be described as. gruesome in its details, nevertheless presents some points of interest.

A primipara, aged 26 years, had been examined on Feb. 27th, 1905, with the object of deciding the approximate date of her confinement. She had been again seen on May 10th with reference to the advisability of calling in $x$ nurse; no examination was made on this oc'casion. A't the: examination in February a vertex presentation was revealed, apparently in all respects normal, foetal heart sounds indieating a living child. 'The husband left for his work' at' 2 P.M.' 